\title{
Modèle de simulation de la durée d'humectation des feuilles au champ après une pluie. Cas d'un couvert homogène
}

Laurent HUBER

INRA, Station de Bioclimatologie, Centre de Recherches de Grignon-Massy-Paris, 78850 Thiverval-Grignon.

\begin{abstract}
La présence d'eau libre à la surface des organes végétaux pendant un temps minimal est un facteur essentiel lors de la contamination de la plante-hôte par un champignon. Afin de prévoir l'occurrence de ce phénomène en terme de durée, un modèle multi-couches de transfert de masse et d'énergie est adapté au cas de la simulation de la durée d'humectation des feuilles après une pluie au sein d'un couvert végétal homogène. Le but est de spatialiser ce paramètre intéressant les phytopathologistes et qui ne fait l'objet actuellement que d'une cstimation globale soit à l'aide d'une mesure standardisée au pare météorologique ou au champ, soit à l'aide d'un modèle simple de bilan d'énergie d'une surface. Nous proposons une paramétrisation originale du mode d'évaporation de l'eau sur les feuilles d'une couche divisée en une zone mouillée et une zone sèche. Les paramètres et variables de la modélisation sont classés au moyen d'une analyse de sensibilité : la capacité d'interception du couvert et son indice foliaire sont 2 paramètres fondamentaux, le mode d'évaporation de l'eau intervient dans une moindre mesure ; la plupart des autres paramètres peuvent être fixés à priori. Les variables externes (rayonnement net, température de l'air, humidité relative, vitesse du vent mesurée à une hauteur de référence au-dessus du couvert sur un pas de temps inférieur ou égal à l'heure) sont déterminantes dans les résultats de la simulation. Une application du modèle montre comment interagissent le couvert et le microclimat; celui-ci permet de prévoir les conditions physiques régnant dans le couvert, caractérisées par de fortes variations verticales éventuelles, en particulicr, en ce qui concerne les durées relatives des épisodes secs et mouillés des différentes strates d'un couvert moyennement à très développé.
\end{abstract}

Mots clés additionnels : Epidémiologie, analyse de sensibilité, simulation.

The persistence of free moisture on leaves for a minimal period of time is very important for the infection of plants by most fungi. In order to predict leaf wetness duration, we propose a one-dimensional multi-layer model of heat and mass transfer in a plant canopy after rainfall. Our aim was to describe the vertical variations of this parameter of interest for phytopathologists. Up till now, it has been measured only at a weather station or calculated from an energy balance model for a single surface without considering any vertical variation. An original aspect consisted in modelling water evaporation on leaves distinguishing wet and dry areas in every layer. We give a classification of the parameters and variables involved in the model according to the sensitivity analysis. The water intercepted by the canopy and the leaf area index were 2 important parameters; water evaporation was less effective and the other parameters could be arbitrarily fixed. The forcing variables (net radiation, air temperature, relative humidity and wind speed measured hourly at a reference height above the canopy) governed the results of the simulation. We stress the value of such a model which takes into account the interactions between canopy and microclimate, and predicts the physical environment in the canopy, with particular regard to interrupted wet periods in different parallel layers of a medium-sized or tall canopy.

Additional key words : Epidemiology, sensitivity analysis, simulation. 


\section{INTRODUCTION}

De nombreux phytopathologistes ont montré l'importance de la persistance d'eau libre à la surface des organes végétaux pour la réussite des phases de germination des spores et de contamination de l'hôte au cours du cycle de la majorité des champignons phytopathogènes aériens (ROTEM, 1978) pour lesquels la présence d'eau joue en tout ou rien; possible en présence d'eau, la contamination est impossible par temps sec. Par ailleurs, l'application des fongicides exige parfois que le couvert soit sec au moment du traitement.

Dans la simulation des épidémies, il est donc nécessaire de pouvoir caractériser la durée d'humectation (des organes sensibles et des feuilles en particulier) : la méthode proposée peut alors être regardée comme un sous-modèle intervenant à certaines étapes du cycle épidémique considéré comme un système à compartiments.

De nombreux auteurs ont proposé des capteurs de durée d'humectation: capteurs mécaniques (NoFFsINGER, 1965), électroniques (HACKEL, 1974; GILLESPIE \& Kidd, 1978 ; Pinguet, 1983; Weiss \& Hagen, 1983; HubAND \& ButLer, 1986), radiométriques (BARTHAKUR, 1985 ; PINTER, 1986). Ces capteurs fournissent une estimation de la durée d'humectation en conditions standard (au parc météorologique ou au champ).

Certains auteurs ont proposé une modélisation de la durée d'humectation à l'échelle du couvert (PAYEN, 1983 ; BUTLER, 1980), sans prendre en considération la variation spatiale de ce facteur, dont on a montré (HUBER, 1987) qu'elle pouvait être importante verticalement (jusqu'à une dizaine d'heures) entre la base et le sommet du couvert ; il a également été montré que les capteurs de durée d'humectation à l'intérieur de la végétation sont d'une utilité limitée dans la mesure des variations spatiales, en raison de la grande variabilité de réponse du capteur imputable à différentes causes (position, orientation, pollution et vieillissement du capteur ; hétérogénéité du milieu).

Afin d'appréhender l'évolution spatio-temporelle de l'humectation dans le couvert, on fait appel à une modélisation unidimensionnelle des transferts de masse et d'énergie. Lemon et al. (1971), Perrier (1976), GOUdRIAAN (1977), NORMAN (1982), CHEN (1984) ont élaboré des modèles globaux de fonctionnement microclimatique du couvert végétal.

Certains de ces auteurs présentent des simulations de l'évolution de la hauteur de rosée, d'autres commentent leurs modèles en énonçant simplement leur utilisation potentielle dans la prévision des durées d'humectation. THOMPSON (1981) et BuTLER (1986) ont utilisé des modèles de simulation de la durée d'humectation (issus de travaux plus anciens: WAGgONER \& REIFSNYDER, 1968 ; CowAN, 1968); le premier ne confronte pas ses résultats avec l'expérimentation, le second se limite à un test sommaire d'un modèle dont la relative complexité réduit l'applicabilité.

L'originalité de notre démarche consiste en l'élaboration d'un modèle relativement simple d'emploi, pour lequel un contrôle expérimental approfondi sur féverole (HUBER, 1987) a permis une validation satisfaisante. Le but de cet article est donc de présenter un modèle, de dégager, par une analyse de sensibilité, des paramètres et variables déterminants, et enfin d'en montrer l'intérêt par une application à une situation météorologique propice à de longues durées d'humectation.

\section{DESCRIPTION DU MODÈLE}

\section{A. Généralités}

Les hypothèses d'un tel modèle micrométéorologique sont classiques (WAGGONER, 1975) :

- homogénéité horizontale du couvert (1 seule variable d'espace),

- on néglige la variation d'énergie stockée du système air-végétation sur de courtes échelles de temps,

- les flux verticaux sont supposés conservatifs audessus du couvert,

- l'air danis les stomates est saturé à la température de surface,

très classiquement, on néglige l'énergie mise en jeu par la respiration et la photosynthèse.

Le schéma numérique consiste à gérer une équation de conservation de l'énergie pour chaque strate horizontale du couvert discrétisé (fig. 1) en adoptant un pas de temps de l'ordre de $1 / 4 \mathrm{~h}$ :

$$
\frac{\partial \mathrm{C}}{\partial \mathrm{t}}=\frac{\partial \phi_{\mathrm{c}}}{\partial \mathrm{z}}
$$

où $\mathrm{C}$ représente la concentration en chaleur ou en vapeur d'eau de la strate considérée et $\phi_{c}$ le flux de cette quantité scalaire. Ce modèle permet de calculer sur chaque pas de temps les températures de surface, de l'air et du point de rosée dans chaque strate.

Pour des raisons de simplicité, et parce que les conditions microclimatiques régnant dans un couvert végétal après pluie par forte nébulosité le justifient, nous avons supposé l'existence d'un régime de convection forcée et de neutralité thermique de l'atmosphère. Les flux verticaux de chaleur sensible et de chaleur latente dans le couvert sont proportionnels aux gradients de température et d'humidité. Le flux $\phi_{\mathrm{c}}$ (c désigne la chaleur ou la vapeur d'eau) s'écrit :

$$
\phi_{\mathrm{c}}(\mathrm{z})=\mathrm{K}(\mathrm{z}) \frac{\partial \mathrm{C}}{\partial \mathrm{z}}
$$

avec la diffusivité turbulente $\mathrm{K}(\mathrm{z})$ s'exprimant à l'aide d'une formulation analytique simple (PERrier, 1967). A partir du profil de diffusivité turbulente, on calcule les résistances aérodynamiques $\operatorname{Ra}(\mathrm{j})(\mathrm{Ra}(\mathrm{j})=\Delta \mathrm{z} / \mathrm{K}(\mathrm{j}))$ entre strates. On suppose l'égalité des diffusivités turbulentes pour la quantité de mouvement, la chaleur et la vapeur.

A l'intérieur d'une strate donnée, les échanges entre l'air et la feuille à l'origine des termes de source sont donnés par:

$$
\phi_{\mathrm{c}}=\mathrm{h}(\mathrm{Cs}-\mathrm{Ca})
$$

où le coefficient d'échange $h$ est une fonction de la vitesse du vent et d'une dimension caractéristique (Clarke \& Wigley, 1975). On suppose l'égalité des coefficients d'échange pour la chaleur et la vapeur. Pour la signification des symboles, on se reportera au tableau 1. 


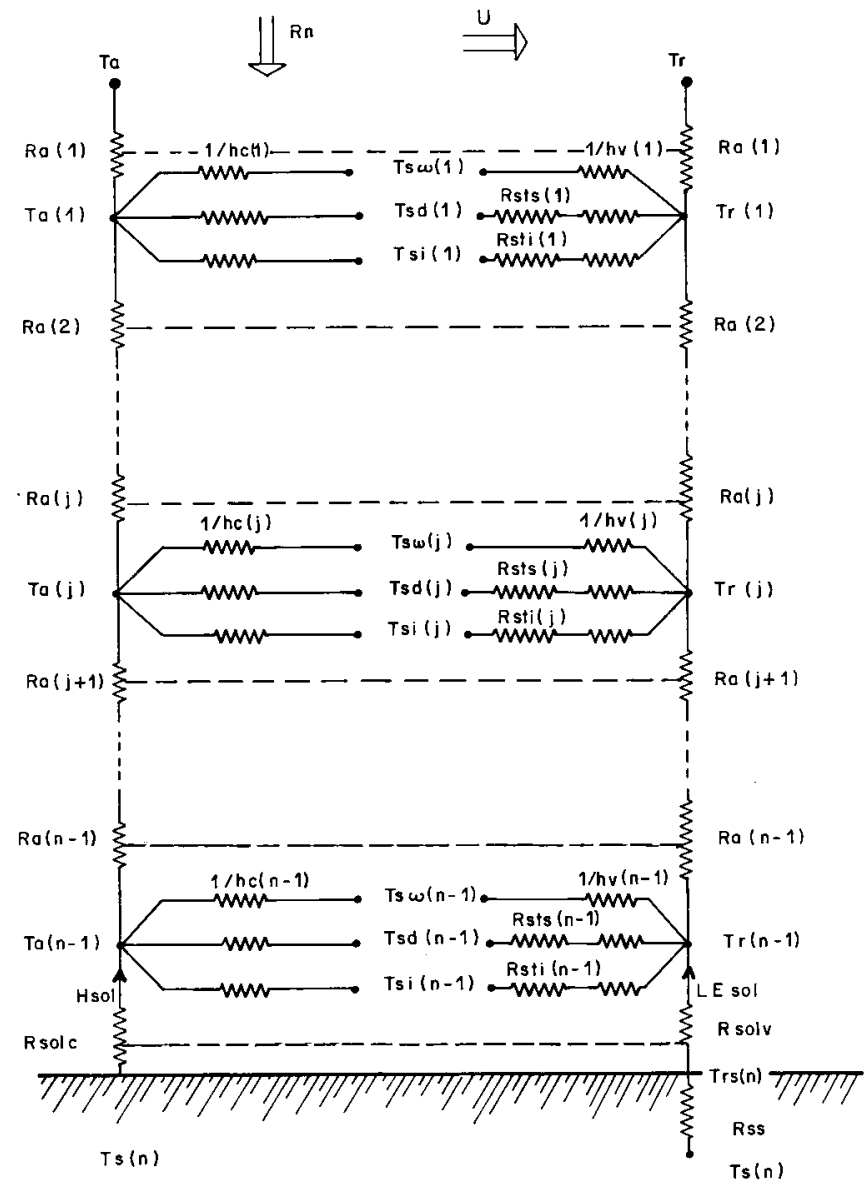

Figure !

Schéma de l'analogie électrique retenue dans la modélisation des transferts de chaleur et de vapeur dans le couvert.

Electrical analogy used in modelling of heat and water vapor transfer in the canopy.

\section{B. Aspects originaux de la modélisation}

Dans chaque strate $\mathrm{j}$ d'indice foliaire LAI(j), on considère 3 zones distinctes qui sont d'une part les surfaces mouillée et non mouillée des faces supérieures des feuilles et d'autre part la surface des faces inférieures des feuilles. Chaque zone est caractérisée par une température de surface (Ts $\omega$, Tsd, Tsi) et contribue aux flux de chaleur sensible et de chaleur latente issus de la strate (on a donc au total 5 profils distincts de température). Le volume d'eau interceptée se répartit sur une aire proportionnelle à $\mathrm{LAI}(\mathrm{j})$ :

$$
\mathrm{Q}(\mathrm{j})=\omega(\mathrm{j}) \cdot \operatorname{LAI}(\mathrm{j}) \cdot \mathrm{e}(\mathrm{j})
$$

où $\omega(\mathrm{j})$ est le facteur de proportionnalité et e(j) l'épaisseur d'eau sur la zone mouillée. La figure 2 illustre les différentes variables employées. Une hypothèse importante consiste à négliger le flux conductif de chaleur entre l'eau libre superficielle et les feuilles elles-mêmes dans une strate considérée; cet aspect est fortement controversé (Butler, 1986; Gillespie \& Duan, 1987).

L'évaporation d'un film d'eau dans une strate consiste en une réduction simultanée de la surface mouillée et de l'épaisseur du film. Dans le cas de gouttes dont le diamètre de base est constant, on a uniquement réduction de la hauteur de la goutte; dans le cas d'un film de surface constante, on a uniquement réduction de l'épaisseur du film. Le processus continu d'évaporation de

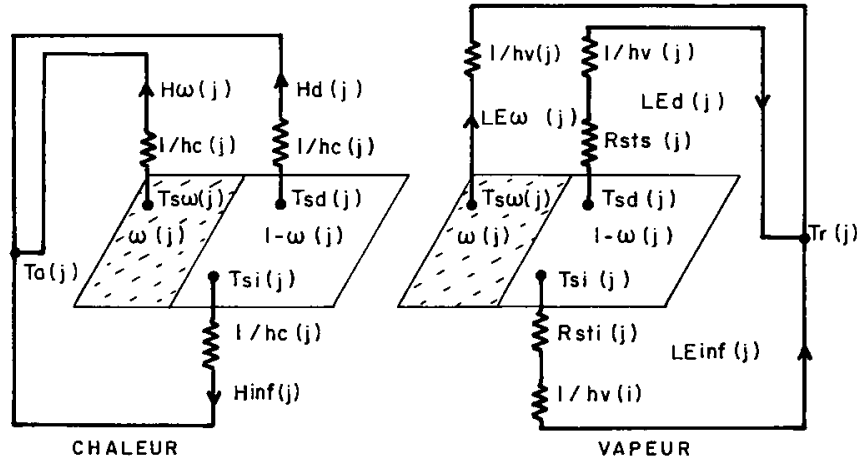

Figure 2

Schéma de l'analogie électrique resemue dans la modélisation des transferts de chaleur et de vapeur à l'échelle d'une strate foliaire (d'indice $i$ ) divisée en 3 zones (surfaces moullée et sèche des faces supérieures des feuilles et surface sèche des faces inférieures).

Electrical analogy used in modelling of heat and water vapor transfer in a foliar layer separated in to 3 sublayers (wet and dry areas of the upper surfaces and dry area of the lower surfaces of leaves).

l'eau sur les feuilles est donc simplifié en supposant que les diminutions de la surface mouillée et de la quantité d'eau présente dans la strate peuvent être imputées à la fin du pas de temps pendant lequel ces 2 grandeurs sont considérées comme constantes.

Des expériences de laboratoire à l'échelle de la feuille ont conduit à une paramétrisation simple du mode d'évaporation de l'eau qui met en relation la quantité d'eau présente sur une feuille et la fraction de surface mouillée de celle-ci.

Les expérimentations ont été effectuées en conditions constantes, mais variables d'une expérience à l'autre $\left(\mathrm{Ta}=24-25^{\circ} \mathrm{C} ; \quad \mathrm{HR}=50-75 \mathrm{p} .100:\right.$ régime de convection libre). Une feuille sectionnée est reliée à un potomètre (KRAMER, 1937) permettant une alimentation hydrique satisfaisante; ce potomètre est placé sur une balance de précision. La feuille est humectée artificiellement par aspersion d'eau conduisant à la présence de gouttes ou de film en fonction du mode d'aspersion. L'ensemble est placé sur une balance ; la balance fournit la cinétique de perte en eau du système par transpiration de la feuille et évaporation de l'eau libre; le capillaire gradué du potomètre donne l'absorption de la feuille. L'hypothèse d'égalité de l'absorption et de la transpiration est bien vérifiée et permet de calculer la quantité Q d'eau libre évaporée en fonction du temps. Par ailleurs, des photographies à la fréquence de 4 à 6 par heure donnent après numérisation et traitement en analyse d'image l'évolution du pourcentage $\omega$ de surface mouillée de la feuille en fonction du temps. On peut donc caractériser la relation entre ces 2 variables $Q$ et $\omega$ pour différents modes d'humectation dẹ la feuille; cette relation est bien représentée par une loi en puissance, transposée à l'échelle de la strate foliaire sous la forme :

$$
\frac{\omega(j)}{\omega_{0}(j)}=\left[\frac{Q(j)}{Q_{0}(j)}\right]^{\beta}
$$

où l'exposant $\beta$ caractérise le mode de répartition de l'eau; $\omega_{0}(j)$ et $Q_{0}(j)$ sont respectivement les valeurs initiales (c'est-à-dire juste au début de la cinétique) de $\omega(\mathrm{j})$ et $Q(\mathrm{j})$. On considère les 2 états extrêmes du mode de répartition de l'eau que sont une humectation en film 
TABLEAU 1

Liste des simboles

C
d
DH
e(j)
$\mathrm{c}_{0}(j)$
$\mathrm{H}$
$\mathrm{h}$

$H d(j), H \omega(j), H i n f(j)$ j

$\mathrm{K}(\mathrm{z})$

LAI

LAI(j)

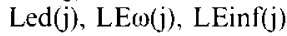

LEsol

$\mathrm{P}^{\prime}$

Ps(T)

Q(j)

Ra(j)

$\operatorname{Rg}(z)$

$\mathrm{Rn}$

$\operatorname{Rs}(z)$

$\mathrm{Rs}_{\min }$

$\mathrm{Rs}_{\max }$

Rsolc, Rsolv

Rss

Rsti(j)

Rsts(j)

$\mathrm{Ta}$

Ta(j)

$\mathrm{Tr}$

$\operatorname{Tr}(\mathrm{j})$

Tsd(j), Tsw(j), Tsi(j)

$\operatorname{Ts}(\mathrm{n})$

U

$\mathrm{U}(\mathrm{z})$

$z$

$\Delta \mathrm{z}$

$\beta$

$\phi$.

$\omega(j)$

$\omega_{10}(\mathrm{j})$ concentration en chaleur ou en vapeur au voisinage d'une surface (Cs) ou dans lair (Ca)

dimension caractéristique des feuilles

durée d'humectation

épaisseur du film d'eau de la zone mouillée

dans la strate $\mathrm{j}$

valeur initiale de $\mathrm{e}(\mathrm{j})$

hauteur du couvert

coefficient d"échange des surfaces pour la chaleur (hc) ou la vapeur (hv)

flux de chaleur sensible issus des 3 zones

de la strate foliaire d'indice $j$

indice rapportant la grandeur

considérée à la strate $\mathrm{j}$

profil de diffusivité turbulente

indice foliaire total du couvert

indice foliaire relatif a la strate $j$

flux de chaleur latente issus des 3 zones

de la strate foliaire d'indice $\mathbf{j}$

flux de chaleur latente issu du sol

pente de la courbe de pression

de vapeur saturante

courbe de pression de vapeur saturante en fonction

de la temperature

volume d'eau dans la strate $\mathrm{j}$

résistance aérodynamique inter-strates

profil de rayonnement global dans le couvert rayonnement net au niveau de référence

résistance stomatique au niveau $z$

résistance stomatique minimale

résistance stomatique maximale

résistances de couche limite au transfert de chaleur

el de vapeur à la surface du sol

résistance au transfert de vapeur à la surface du sol

résistance stomatique des faces inférieures

des feuilles de la strate $\mathrm{j}$

résistance stomatique des faces supérieures

des feuilles de la strate $\mathrm{j}$

température de l'air au niveau do référence

température de l'air dans la strate j

température du point de rosée de l'air

au niveau de référence

température du point de rosée de l'air

dans la strate $j$

températures de surface des 3 zones

de la strate foliaire d'indice $j$

température de surface du sol

variable temporelle

vitesse du vent au niveau de référence

profil de vitesse moyenne du vent dans le couvert

hauteur au-dessus du sol

ćpaisseur de chaque strate

exposant dans la relation entre volume d'cau

et surface mouillée

densité de flux du scalaire $\mathrm{C}$

proportion de surface mouillée des faces supérieures

des feuilles de la strate j

valeur initiale de $\omega(\mathrm{j})$ heat and vapor concentration near a surface

$(\mathrm{Cs})$ or in the air (Ca)

leal characteristic dimension

leaf wetness duration

water film thickness

of the wet area in layer $j$

initial value of $e(j)$

canopy height

heat (hc) and vapor (hv)

exchange coefficients

sensible heat flux densities emanating from

the 3 sublayers of the foliar layer $j$

index referring to the foliar layer

turbulent difrusivity

global leaf area index

leaf area index for layer $\mathrm{j}$

latent heat flux densitics emanating

from the 3 sublayers of the foliar layer $j$

latent heat flux density emanating from the soil

slope of the saturated vapor pressure curve

salurated vapor pressure at temperature $\mathbf{T}$

water volume in layer $j$

aerodynamic resistance along

the vertical direction

global radiation profile in the canopy

net radiation at reference height

stomatal resistance at height $\mathrm{z}$

minimum stomatal resistance

maximum stomatal resistance

boundary layer resistances for heat and vapor transfer near the soil

resistance to vapor transfer emanating from the soil

leaf stomatal resistance of lower surfaces in layer $\mathrm{j}$

leaf stomatal resistance of upper surfaces in layer $\mathrm{j}$

air temperature at reference height

air temperature in layer $j$

dew-point temperature at reference height

dew-point temperature in layer $j$

surface temperatures of the 3 sublayers

in foliar layer $\mathrm{j}$

soil surface temperaturc

time

windspeed at reference height

windspeed at height $z$

height above the soil

layer thickness

exponent in the power law beetwen water volume

and wet area

fux density of scalar $\mathrm{C}$

wet area fraction of upper surfaces of layer $j$

initial value of $\omega(\mathrm{j})$ de surface constante $(\beta=0)$ ou d'épaisseur constante $(\beta=1)$ et une humectation en gouttes cylindriques $(\beta=0)$ ou hémisphériques $(\beta=2 / 3)$. La figure 3 donne les résultats obtenus au laboratoire (HUBER, 1987) pour l'évolution du rapport $\omega / \omega_{0}$ en fonction de $\mathrm{Q} / \mathrm{Q}_{0}$ lorsque l'on a affaire à des films ou des gouttes sur une feuille isolée et en conditions normales d'alimentation hydrique. L'absence de convection forcée ne nuit pas à l'expérimentation dans la mesure où le but était uniquement de créer des conditions variables de surface mouillée et de quantité d'eau présente sur une feuille.

\section{Paramétrisations}

Les variables indépendantes de l'état d'humectation de la surface, que sont la vitesse du vent et l'extinction des rayonnements net et global (le rayonnement net est partiellement fonction de la température de surface des feuilles), sont calculées à partir de sous-modèles autonomes directement au moyen des variables de forçage et conformément à des résultats de la littérature. A l'échelle de temps du phénomène d'humectation (de l'ordre de quelques jours au maximum), le profil d'indice foliaire est supposé constant. 


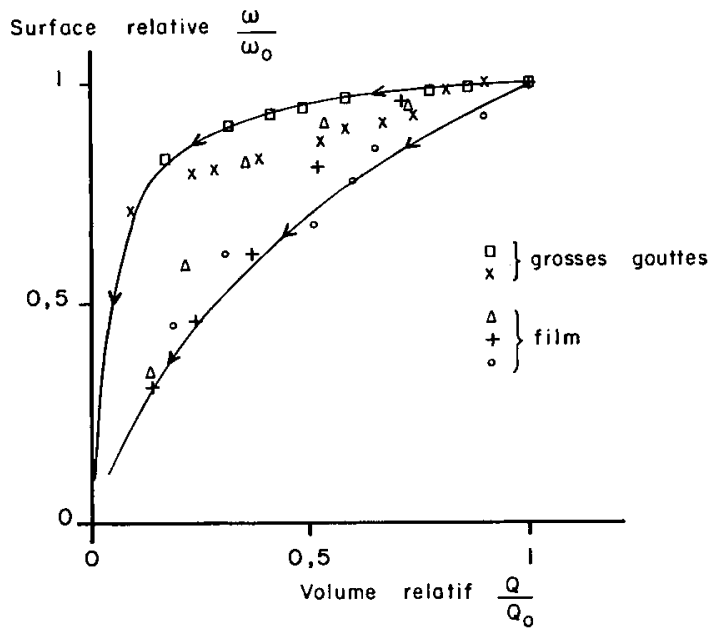

Figure 3

Courbes d'évolution (tomps: sens de la fleche) de la surface mouillée d'une feuille (rapportée à la surface initialement mouillée $\left(\omega_{t}\right)$ en fonction du volume d'eau au même instant (rapporté au volume d'eau intitial $Q_{0}$ ) pour différents modes d'évaporation (film ou gouttes).

The ratio of remaining wet area $\omega$ to initial wet area $\omega_{0}$ as a function of the ratio of remaining intercepted water volume $Q$ to intial intercepted water volume $Q$. These laboratory measurements show different parametrizations of water evaporation

(films or drops) for five experiments

versus time (time : arrow direction).

Pour la vitesse moyenne du vent, on a un profil logarithmique classique au-dessus du couvert; dans le couvert, on retient l'allure proposée par LANDSBERG \& JAMES (1971) par application du modèle de THOM (1971) pour le calcul du profil de diffusivité turbulente. Pour les rayonnements net et global, on retient une extinction exponentielle (IMPENS \& LEMEUR, 1969 ; FASHEUn \& DENNETT, 1982).

Les résistances de couche limite au niveau des feuilles s'expriment à l'aide d'une fonction affine de la variable $[\mathrm{d} / \mathrm{U}(\mathrm{z})]^{1 / 2}$ (FINNIGAN \& RAUPACH, 1987).

Les résistances stomatiques sont données par l'équation suivante (AvissARet (al., 1985) :

$$
\frac{1}{R s(z)}=\frac{1}{R s_{\text {max }}}+\left[\frac{1}{R s_{\text {min }}}-\frac{1}{R s_{\text {max }}}\right] * f(R g(z))
$$

où $\mathrm{Rs}_{\max }$ et $\mathrm{Rs}_{\min }$ sont respectivement les résistances stomatiques maximale et minimale des zones sèches des feuilles, $f(\operatorname{Rg}(z))$ est une fonction linéaire du rayonnement global incident dans la strate $\mathrm{j} \cdot \mathrm{Rs}_{\max }, \mathrm{Rs}_{\min }$, $f(\operatorname{Rg}(z))$ ont des expressions différentes pour les faces inférieures et supérieures des feuilles. La résistance stomatique des surfaces mouillées est supposée nulle.

Le flux de chaleur latente issue du sol est analogue à un flux de transpiration : on introduit une résistance Rss de type stomatique dont là valeur est fixée en fonction de l'humidité de surface du sol (ShutTleworTh \& WALLACE, 1985).

\section{Schéma de calcul. Conditions aux limites. Conditions initiales et finales}

L'écriture du bilan d'énergie et des lois d'Ohm relatives aux transferts de chaleur et de vapeur pour les 3 zones de chacune des différentes strates aboutit à un système linéaire que l'on peut résoudre par inversion matricielle (Furnival et al., 1975). CHEN (1981) propose une méthode itérative plus élégante et économe en temps de calcul que nous avons adaptée à notre cas.

Les variables de forçage (variables climatiques mesurées nécessaires au fonctionnement du modèle) sont les températures de l'air et du point de rosée ainsi que la vitesse moyenne du vent et le rayonnement net. Ces variables sont mesurées à un niveau de référence audessus du couvert.

On suppose que le couvert est saturé d'eau postérieurement à une pluie; la hauteur d'eau interceptée par l'ensemble du couvert est répartie dans les différentes strates proportionnellement à leurs indices foliaires respectifs. L'épaisseur initiale $e_{0}$ du film d'eau équivalent sur les feuilles est pris égal à $0,07 \mathrm{~mm}$ (l'interception nette d'un couvert de LAI égal à 7 est alors d'environ $0,5 \mathrm{~mm}$ ). On prend pour $\omega_{0}(\mathrm{j})$, fraction d'indice foliaire mouillée à l'instant initial, la valeur de 0,95 (couvert quasi uniformément humecté en film dans le cas de la féverole); BUTLER (1986) propose une valeur de 0,1 pour cette variable dans le cas du blé où l'humectation est sous forme de gouttes en raison de la nature hydrophile de la cuticule.

Pendant le pas de temps t, on suppose l'existence d'un régime permanent des échanges; à la fin du pas de temps, on impute le flux d'évaporation calculé dans chaque strate sous la forme d'une diminution du stock d'eau Q(j). Lorsque le calcul donne un stock d'eau résiduelle nul dans la strate $\mathrm{j}$, on en déduit la durée d'humectation relative à cette strate. Pour une pluie donnée, le modèle cesse de tourner lorsque la dernière strate encore mouillée voit son bilan d'eau superficielle annulé (la plupart du temps, cette dernière strate se situe à la base du couvert).

\section{ANALYSE DE SENSIBILITÉ}

Cette analyse a été effectuée avec des conditions aux limites correspondant à des valeurs couramment rencontrées lors de situations propices à de longues durées d'humectation : humidité relative élevée, rayonnement net faible, vitesse du vent faible. Notons que, lorsque la vitesse du vent dans le couvert est particulièrement basse $\left(<0,2 \mathrm{~m} \cdot \mathrm{s}^{-1}\right)$, nous nous trouvons en limite d'application du modèle, en raison de l'existence éventuelle d'un régime de convection libre. Le tableau 2 donne la gamme, observée lors des expérimentations, des variables météorologiques de forçage du modèle, ainsi que les valeurs standard retenues pour l'analyse de sensibilité.

La valeur de $\beta$ est fixée à $1 / 3$, ce qui correspond à une diminution du volume d'eau libre dans chaque strate qui privilégie la réduction en épaisseur par rapport à la réduction de surface.

Pour le couvert, on retient un LAI de 6,8 réparti en tranches d'épaisseur égale à $0,2 \mathrm{~m}$; de bas en haut, on a 7 strates de végétation $(\mathrm{H}=1,4 \mathrm{~m})$ de densité de surface foliaire: $3.3 ; 3,9 ; 4,8 ; 5.4 ; 6.6 ; 5,8 ; 4,4$ (valeurs mesurées). La densité de surface foliaire d'une strate est donnée par le rapport de l'indice foliaire à l'épaisseur de la strate. 
TABLEAU 2

Gamme de variation des variables de forcage et valeurs retemues dans l'analyse de sonsibilité.

Variation range of the forcing variables and standard values used in the sensitivity analvis.

\begin{tabular}{lcc}
\hline \hline Variable & Gamme de variation & Valeur standard \\
\hline $\mathrm{Rn}\left(\mathrm{W} \cdot \mathrm{m}^{2}{ }^{2}\right.$ & $50-300$ & 200 \\
$\mathrm{U}\left(\mathrm{m} \cdot \mathrm{s}^{1}\right)$ & $0-5$ & 1.5 \\
$\operatorname{Ta}\left({ }^{\circ} \mathrm{C}\right)$ & $8-20$ & 15 \\
$\operatorname{Tr}\left({ }^{\circ} \mathrm{C}\right)$ & $5-15$ & 12 \\
$\mathrm{HR}(\%)$ & $60-100$ & 80 \\
\hline \hline
\end{tabular}

\section{A. Sensibilité aux variables météorologiques}

Les figures $4 a, b, c$ illustrent l'influence de variation des variables de forçage (au nombre de 4); le principe consiste à fixer 3 de ces variables en faisant varier la quatrième.

A $40 \mathrm{~cm}$ au-dessus du sol, la durée d'humectation des feuilles augmente de 50 p. 100 lorsque le rayonnement net ou la vitesse du vent au niveau de référence diminuent de moitié (fig. $4 a$ et $4 b$ ). Les rôles respectifs des températures de l'air $\mathrm{Ta}$ et du point de rosée $\mathrm{Tr}$ sont symétriques: c'est la différence $(\mathrm{Ta}-\mathrm{Tr})$ qui est déterminante des variations de la durée d'humectation (fig. $4 \mathrm{c}$ ) ou si l'on préfère l'humidité relative. A $40 \mathrm{~cm}$, si $(\mathrm{Ta}-\mathrm{Tr})$ passe de 5 à $1^{\circ} \mathrm{C}$, la duré d'humectation calculée est multiplée par un facteur voisin de 2.

\section{B. Influence des variations de divers paramètres}

\section{Paramètres relatifs à l'humectation}

Selon la valeur de l'épaisseur $e_{0}$ du film d'eau, on obtient de grandes variations de la durée d'humectation calculée, et d'autant plus qu'il s'agit d'une strate proche du sol (fig. 5a). La durée d'humectation est légèrement plus que proportionnelle à $\mathrm{e}_{\mathrm{j}}$, toutes conditions égales par ailleurs. Cette épaisseur $\mathrm{e}_{0}$ est donc un paramètre crucial de la modélisation.

Le mode d'évaporation de l'eau (gouttes ou films' est, dans une moindre mesure, à l'origine de variations possibles de la durée d'humectation. La figure $5 \mathrm{~b}$ montre comment le choix de la valeur de $\beta$ influe sur le résultat de la simulation, les autres paramètres étant constants; il existe un facteur voisin de 2 entre une évaporation en film constamment continu $(\beta=0)$ et une évaporation de portions d'un film discontinu ou de gouttes pour lesquelles surface et épaisseur décroissent simultanément ( $\beta=0,7$ par exemple). Les simulations effectuées supposent une valeur unique de $\beta$, mais il n'est pas exclu d'envisager sa variation spatiale: par exemple, la mouillabilité des feuilles jeunes du sommet du couvert entraîne parfois la présence de gouttes à leur surface tandis que les feuilles âgées de la base sont humectées par un film d'eau.

\section{Paramètres relatifs au couvert}

Pour un indice foliaire LAI dont la valeur double ( 3,4 à 6,8 ), la durée d'humectation calculée à $40 \mathrm{~cm}$ est
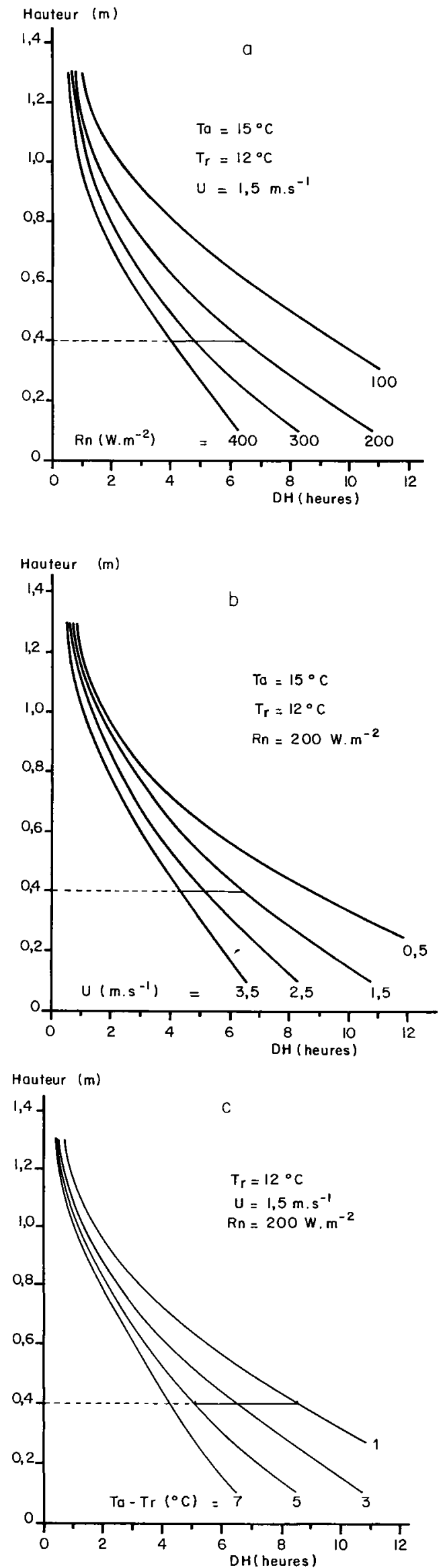

Figure 4

Sensibilité du modèle aux variables de forçage: rayonnement net $R n$ $(a)$, vitesse du vent $U(b)$, différence entre temperatures de l'air Ta et du point de rosée de l'air Tr (c). (DH : durée d'humectation calculée).

Variation in wetness duration profile as a function of variation in forcing variables: (a) net radiation $R n$ and (b) wind speed $U$, (c) air temperature Ta minus dew-point temperature Tr. 

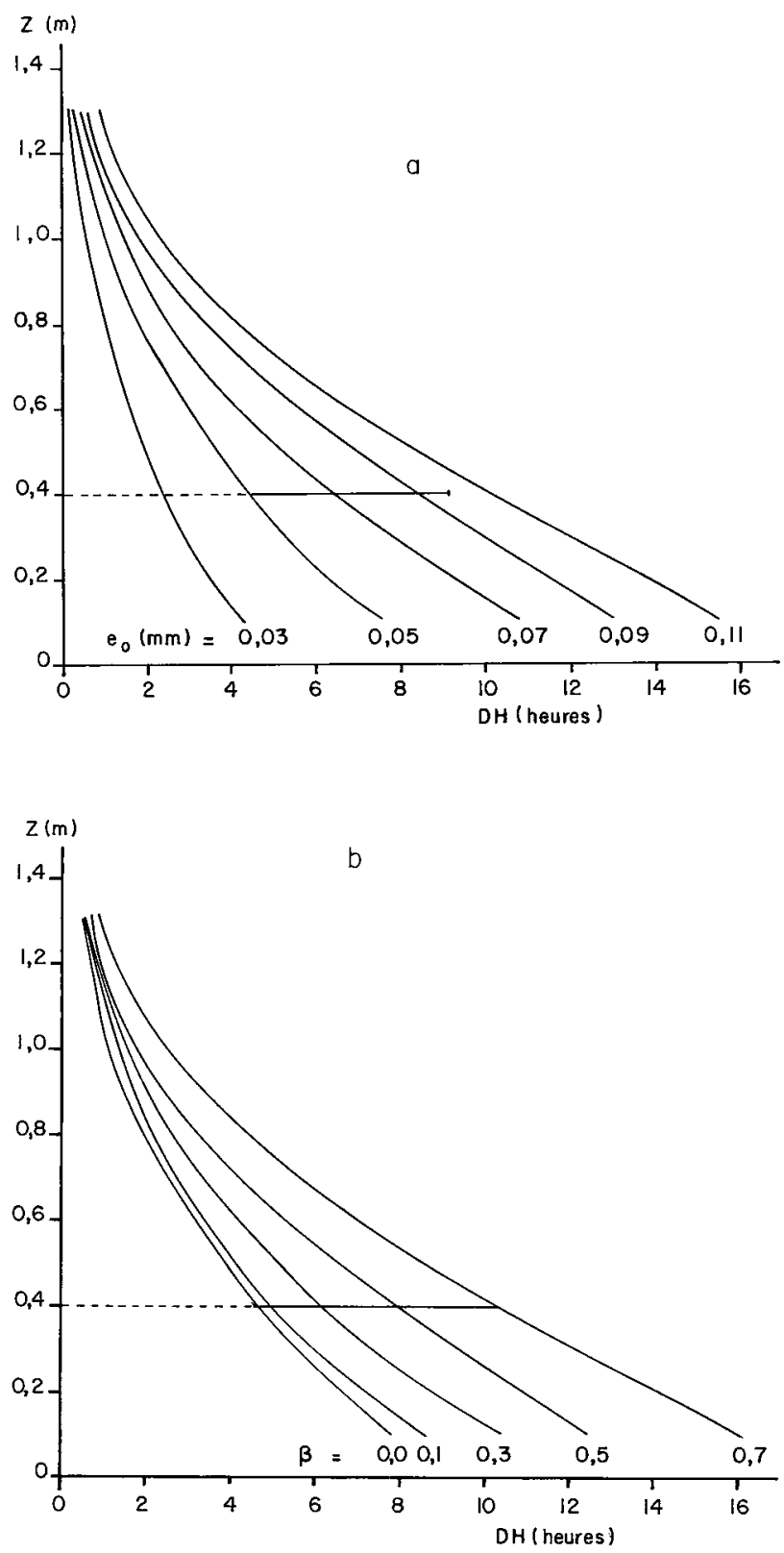

Figure 5

Sensibilité du modèle aux paramètres de l'humectation: épaisseur initiale $e_{0}$ du film d'eau (a) et mode d'évaporation de l'eau (film ou gouttes) paramétrisé à l'aide du facteur $\beta(b)$.

Variation in wetness duration profile as a function of variation in water deposit parameters: initial water film thickness $e_{0}$ (a) and water evaporation parameter $\beta$ (b) for water films or drops.

multipliée par 3 pour une même allure de la fonction de densité de surface foliaire (fig. 6a). Ce paramètre joue à la fois sur la valeur du volume Q(j) d'eau interceptée dans la strate $\mathrm{j}$ proportionnel à LAI(j), et sur l'extinction des rayonnements net et global et de la vitesse du vent qui déterminent l'intensité des échanges. Tout autant que $\mathrm{e}_{0}$, le paramètre LAI est à connaître avec une précision suffisante.

De manière moins nette, la forme de la courbe de densité de surface foliaire a une influence sur le profil simulé de la durée d'humectation (fig. 6b) ; on observe un écart maximal de $2 \mathrm{~h}$ entre les 2 courbes correspondant à une situation du maximum de densité de surface foliaire en haut ou en bas du profil. Aux 2 bornes du
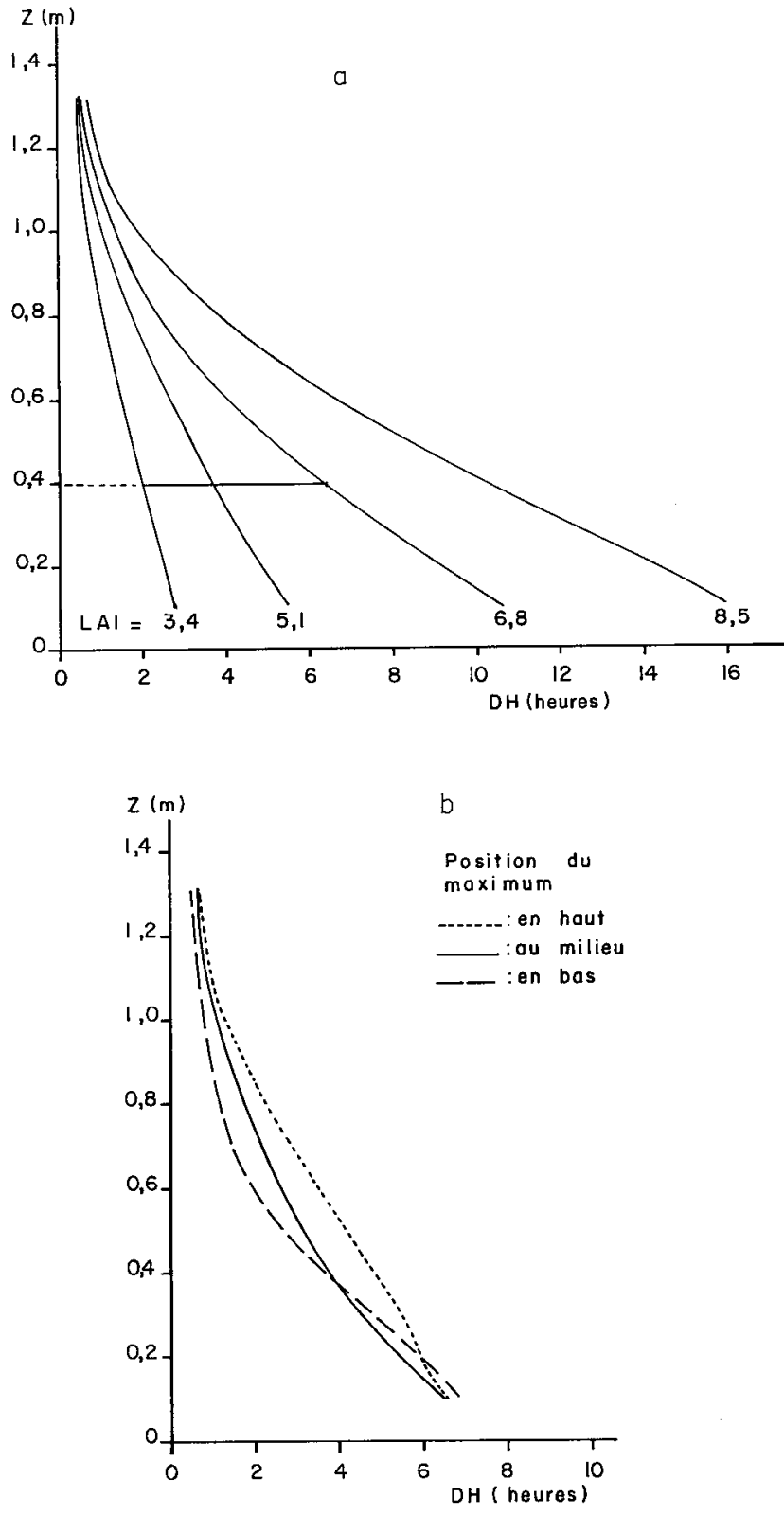

Figure 6

Sensibilité du modèle aux paramètres du couvert : indice foliaire total LAI (a) et distribution de densité de surface foliaire (b): 3 cas différenis en fonction de la position du maximum de la distribution pour un LAI constant égal à 5,6 .

$V$ Variation in wetness duration profile as a function of variation in canopy parameters: leaf area index $L A I$ (a) and leaf area density (b) for 3 different cases as a function of the position of the leaf area density maximum $(L A I=5,6)$.

couvert, les durées d'humectation calculées ne diffèrent pas selon l'allure de cette fonction de densité.

De ces différentes remarques, on conclut que la mesure de surface foliaire par strate n'est pas indispensable; il suffit d'allier la connaissance de l'allure théorique de cette courbe avec les mesures de la hauteur $\mathrm{H}$ et de l'indice foliaire LAI du couvert.

Il convient d'insister sur une limitation de la modélisation proposée dans la mesure où celle-ci ne prend pas en compte l'influence du poids de l'eau sur les feuilles pouvant déterminer une modification de la géométrie du couvert avec une conséquence possible sur la valeur du coefficient d'extinction. Toutefois, l'analyse de sensibilité montre qu'une variation de \pm 40 p. 100 de ce 
coefficient (dont la valeur est fixée à 0,5 dans les simulations) n'entraîne qu'une faible variation $( \pm 3$ p. 100$)$ de durée d'humectation de la strate inférieure; il faut conclure, dans les situations qui nous intéressent (faible rayonnement), que c'est surtout le transfert de chaleur sensible en chaleur latente qui est responsable de l'évaporation de l'eau libre, ce qui est tout à fait cohérent avec les valeurs très faibles calculées pour le rayonnement net dans les strates basses du couvert.

\section{Autres paramètres ou paramétrisations}

Certaines paramétrisations jouent également sur les résultats de la simulation, mais de manière telle que la caractérisation expérimentale de celles-ci n'est pas nécessaire. On a ainsi testé (Huber, 1987) l'influence de la valeur de diverses résistances, ou du profil de diffusivité turbulente (fig. 7) pour aboutir à la conclusion que l'introduction de valeurs plausibles est suffisante.

\section{APPLICATION DU MODÈLE À UNE SITUATION MÉTÉOROLOGIQUE PROPICE À DE LONGUES DURÉES D'HUMECTATION}

\section{A. Conditions de la simulation}

Sur une période de 6 jours consécutifs (PlaisirGrignon; 16-21/06/87) pendant lesquels de fréquentes pluies ont entraîné des durées d'humectation importan-

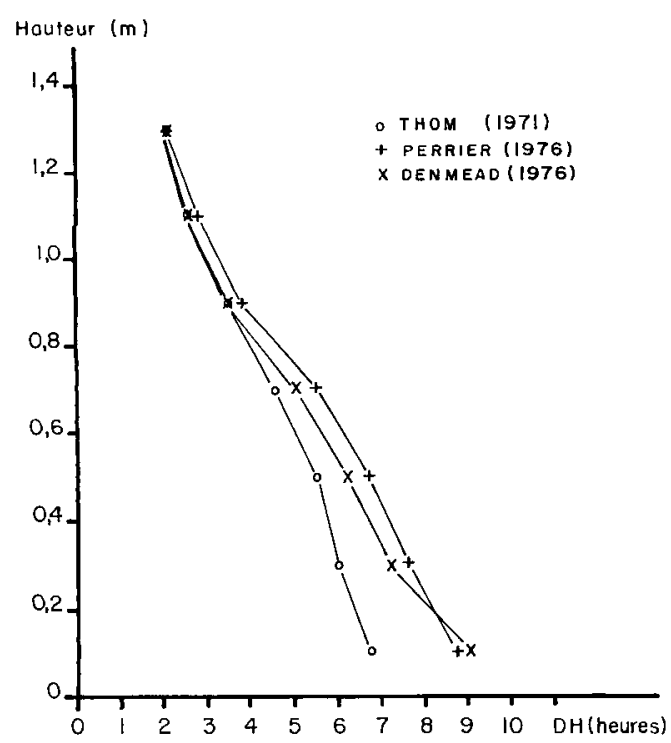

Figure 7

Sensibilité du modèle au choix de la forme du profil de diffusivité turbulente 3 différentes expressions analytiques issues de la bibliographie).

Variation in wetness duration profile as a function of variation in turbulent diffusivity profile (three different analytical expressions obtained from the bibliography).
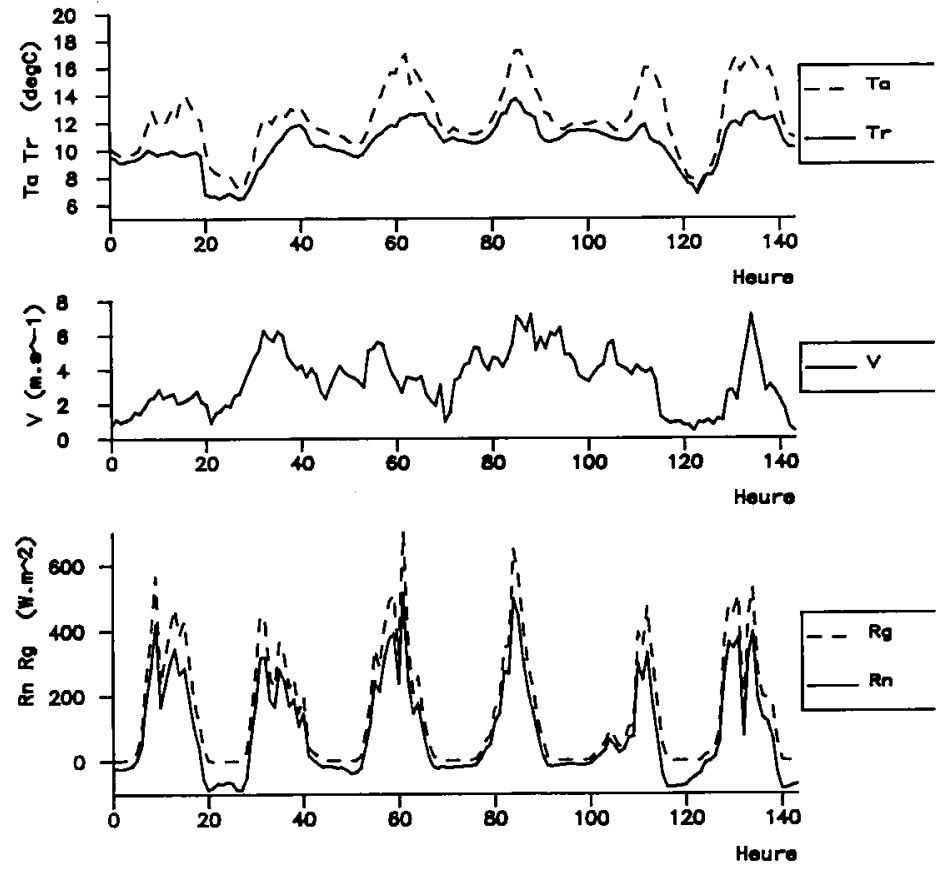

Figure 8

Evolution des variables de forcuge du modele durant la période de simulation (16-2l juin 1987).

Time traces of external variables during the simulation period (I6th$21.51 / 06 / 87)$

tes, on a fait tourner le modèle pour simuler l'évolution de l'eau libre à la surface des feuilles. Les mesures utilisées ont été effectuées sur un pas de temps d'une heure (fig. 8).

Pour une telle simulation, il convient de répartir la lame d'eau interceptée dans les $(\mathrm{n}-1)$ strates de végétation, sachant qu'au-delà de la capacité d'interception, l'eau incidente dans une strate est redistribuée par égouttement sur les strates sous-jacentes. Lorsque le couvert est totalement saturé, tout supplément de précipitation est fourni au sol. Pour modéliser cette répartition, on a utilisé un schéma analogue à celui présenté par RUTTER \& MORTON (1977) pour la modélisation de l'interception des forêts. Les simulations ont été effectuées avec les valeurs standard des paramètres d'humectation et du couvert.

\section{B. Résultats et discussion}

La figure 9 montre comment la hauteur du couvert influe sur la durée d'humectation des différentes strates, et particulièrement de la strate inférieure du couvert.

On constate que le nombre de périodes continues d'humectation de la strate inférieure passe de 7 dans le cas d'un couvert de $0.4 \mathrm{~m}$ de hauteur à 1 dans le cas d'un couvert de $1,4 \mathrm{~m}$. Pour la strate $20-40 \mathrm{~cm}$, on passe de 9 périodes distinctes à 5 . Cette simulation montre au pathologiste qu'une caractérisation au laboratoire de la durée minimale d'humectation pendant la phase de contamination d'un agent pathogène foliaire n'est pas facilement transposable au champ.

Lorsque la hauteur de pluie est faible, il y a peu de différence entre strates, principalement en raison du fait que ce sont les strates supérieures qui interceptent la majeure partie de l'eau incidente et, en même temps, la 
plus grande part du rayonnement net, tandis que les strates inférieures peu humectées sont sèches dans un délai analogue, voire plus tôt. On voit donc comment interagissent la distribution temporelle des précipitations et la hauteur du couvert dans l'occurrence de l'humectation que l'on doit analyser en terme de durée des épisodes avec humectation, mais aussi de durée des épisodes d'interruption de l'humectation, sachant qu'un inoculum ayant commencé à germer va se trouver fortement pénalisé, voire détruit par un épisode sec (EISENSMith et al., 1982 ; Olivier et al., 1983).

\section{CONCLUSIONS, PERSPECTIVES}

L'analyse de sensibilité permet de hiérarchiser l'importance des différentes variables et des paramètres retenus dans la modélisation. Les facteurs discriminants de la durée d'humectation sont, par ordre décroissant: l'indice foliaire LAI, la hauteur maximale d'eau interceptée $\left(\mathrm{LAI} \cdot \mathrm{e}_{0}\right)$, la différence entre les températures de l'air Ta et du point de rosée Tr (ou encore l'humidité relative de la masse d'air) au niveau de référence, puis la vitesse moyenne du vent. Viennent ensuite le mode de résorption de l'eau superficielle, la résistance stomatique, la résistance au transfert de vapeur depuis le sol, le profil de diffusivité turbulente (les 3 derniers facteurs intervenant faiblement sur la sensibilité du modèle).

Nous avons vu un cas d'application du modèle dans la recherche des intervalles humectés et secs, sachant que ce sont leurs durées relatives qui doivent être considérées vis-à-vis du risque épidémique et que la spatialisation du phénomène d'humectation ne peut en aucun cas être négligée. On a ici l'occasion de souligner que les conditions microclimatiques du couvert résultent d'une interaction plante-climat, et que c'est donc au sein du pathosystème hôte-climat-parasite qu'il convient de situer la simulation des épidémies, en ayant à l'esprit que l'on ne pourra pas correctement caractériser l'environnement des agents pathogènes à partir des données mésoclimatiques du parc météorologique.

D'autres applications du modèle peuvent être envisagées comme la gestion de l'irrigation par aspersion de manière à définir les périodes d'irrigation en minimisant les risques phytosanitaires; il est également possible d'effectuer une analyse fréquentielle (zonage agroclimatique) de l'occurrence des longues durées d'humectation, comparativement à une méthode beaucoup plus approximative couramment employée qui consiste à utiliser un critère-seuil sur l'humidité relative au parc météorologique.

Ce modèle présente l'avantage d'une relative simplicité alliée à une démarche déterministe; toutefois, il possède certaines contraintes comme la mesure de paramètres du couvert ou la valeur du pas de temps (on doit disposer de mesures des variables de forçage sur un pas de temps inférieur ou égal à l'heure). Ce modèle doit faire l'objet d'une adaptation à différentes cultures, il est en effet évident que son utilisation en routine dans l'état actuel ne sera pas vraiment satisfaisant dans la mesure où celui-ci considère l'homogénéité horizontale du couvert, ce qui constitue une limitation importante, étant donnée la très forte variabilité de l'architecture et de la mouillabilité d'un couvert à un autre, cette variabilité n'étant pas sans conséquence sur l'interception et donc sur la durée d'humectation.

Par ailleurs, ce modèle requiert des données météorologiques enregistrées de telle manière que l'on pourra considérer l'évolution temporelle des variables indépendantes de la surface (rayonnement global, pluie) identique au niveau de la parcelle et du site de mesure. L'utilisation pratique du modèle suppose donc l'existence d'une station agroclimatique automatique au

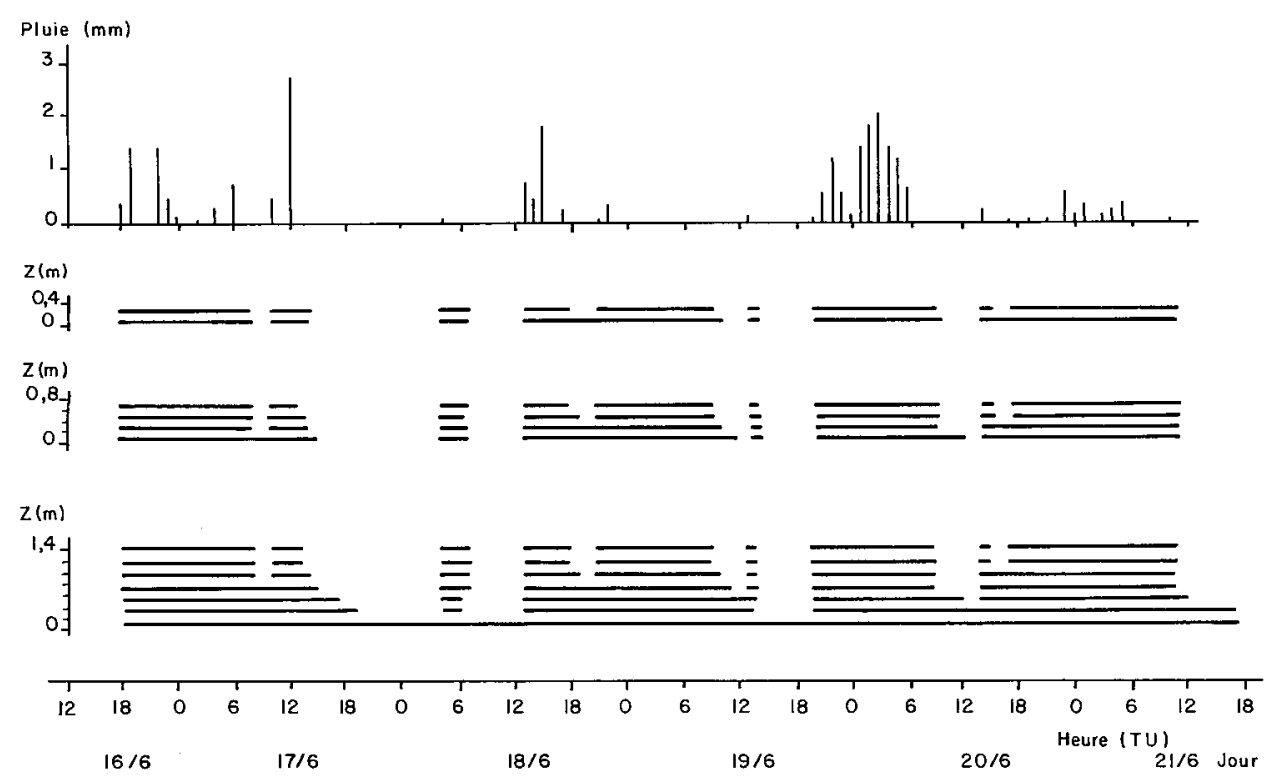

Figure 9

Simulation du profil de durée d'humectation d'un couvert de féverole pour différentes hauteurs du couvert sur une période de 6 jours (16-21 juin 1987) caractérisé par de fréquentes précipitations (le nombre de strates de $0,2 \mathrm{~m}$ d'épaisseur varie de 1 à 7 et le LAI de 0,6 à 6,$4 ;$ les données météorologiques utilisées sont des moyennes horaires).

Variation in simulated leaf wetness duration profile as a function of canopy height during a 6-day period (I6th-21st/06/87) with frequent rain (layer number: 1 to 7 ; LAI: 0.6 to 6.4 ; the meteorological data are hourly averages). 
niveau d'un groupement de producteurs ou encore de l'exploitation agricole elle-même afin de s'abstraire des problèmes liés à la forte variabilité spatiale des variables précitées. A moyen terme, ce type de station sera économiquement abordable; l'acquisition des données et leur traitement en temps réel par un logiciel adéquat constituent une perspective raisonnable, le modèle proposé étant alors un des modules d'un logiciel plus général d'aide à la décision.

Recule 25 fëvrier 1988. Accepté le 29 mai 1988.

\section{RÉFÉRENCES BIBLIOGRAPHIQUES}

Avissar R., Avissar P., Mahrer Y., Bravdo B. A., 1985. A model to simulate response of plant stomata to environmental conditions. Agric. For. Meteorol., 34, 21-29.

Barthakur N. N., 1985. A comparative study of radiometric and electronic leaf wetness sensors. Agric. For. Meteorol., 36, 83-90)

Butler D. R., 1980. Dew and thermal fog: measurements and an estimate of wetness duration on cocoa pods. Quart. J. Roy. Metcorol. Soc., 106, 539-550.

Butler D. R., 1986. Evaporation from rain drops on leaves in a cereal canopy: a simulation model. Bound. Laver Meterol., 36, 39-51

Chen J., 1981. Uncoupled multi-layer model for the transfer of sensible and latent heat flux densities from vegetation. Bound. Laver Meteorol., 28, 213-228.

Chen J., 1984. Mathematical analysis and simulation of crop micrometeorology. Thesis, Wageningen, $116 \mathrm{p}$.

Clarke J. A., Wigley G., 1975. Heat and mass transfer from real and model leaves. In: "Heat and mass transfer in the hiosphere ", part I. do D. A. Vries \& N. H. Afgan ed., 413-422.

Cowan I. R., 1968. Mass, heat and momentum exchange between stands of plants and their atmospheric environment. Quart. J. Roval Meteorol. Soc.. 94, 523-544.

Denmead O. T., 1976. Temperate cercals. In: "V'egetation and the atmosphere", vol. II, J. L. Monteith ed., Academic Press, London, $1-31$.

Eisensmith S. P., Jones A. L., Cress C. E., 1982. Effects of interrupted wet periods on infection of sour cherry by Cocomnces hicnalis. Phyropathology, 72, 680-682.

Fasheun A., Dennett M. D., 1982. Interception of radiation and growth efficiency in field beans (Vicia faba L.), Agric. Meteorol. 26. $221-229$.

Finnigan J. J., Raupach M. R., 1987. Transfer process in plant canopies in relation to stomatal characteristics. In : "Stomotal function » E. Zeiger, G. Farquhar, I. Cowan ed., Standford Univ. Press.

Furnival G. M., Waggoner P. E., Reifsnyder W. E., 1975. Compliting the energy budget of a leaf canopy with matrix algebra and numerical integration. Agric. Meteorol., 14, 405-416.

Gillespie T. J., Kidd G. E., 1978. Sensing duration of leaf moisture retention using electrical impedance grids. Can. J. Plant $S_{c} i .58$ 179-187.

Gillespie T. J., Duan R. X., 1987. A comparison of cylindrical and that plate sensors for surface wetness duration. Agric. Forest Meteorol. 40, $61-70$.

Goudriaan J., 1977. Crop micrometeorology : a simulation study Simulation monographs, Pudoc, Wageningen, $249 \mathrm{p}$.

Hackel H., 1974. Eine elektrische Methode zur Messung der Blattbenetzungsdauer unmittelbar am Blatt. Agric. Meteorol., 13 (1). 91-103.

Huband N. D. S., Butler D. R., 1984. A comparison of wetness sensors for use with computer or microprocessor systems designed for disease forecasting. Proc. British crop protection conference "Pests and diseases », vol. II, 633-638.

Huber L., 1987. Modélisation spatio-temporelle de la durée dhumectation d'un couvert végétal après une pluie. Thèse Doc. Ing., INA P-G, $156 \mathrm{p}$.
Impens I., Lemeur R., 1969. Extinction of net radiation in different crop canopies. Arch. Met. Geoph. Biokl., ser. B., 17, 403-412.

Kramer P. J., 1937. The relation between rate of transpiration and rate of absorption of water in plants. Ann. J. Bot., 124, 10-15.

Landsberg J. J., James G. B., 1971. Wind profiles in plant canopics studies on an analytical model. J. Appl. Ecol., 8, 729-741.

Lemon E., Stewart D. W., Shawcroft R. W., 1971. The sun's work in a cornficld. Science. 174, 371-378.

Noffsinger T. L., 1965. Survey of techniques for measuring dew. In : "Humidity and moisfure measurement and control in science and industry" vol. 2, A. Wexler ed., Reinhold Publ. crop. New York. 523-531

Norman J. M., 1982. Simulation of microclimates. In : "Biometeorologry in integrated pest management", J. Hatfield \& I. Thomason ed. 65-69. Academic Press, New York.

Olivier J. M., Lambert C., Lefeuvre M., 1983. Application du thermohumectographe KIT-INRA. Etude des risques de tavelure du pommier à l"échelle du Maine-et-Loire (France). Bull. OEPP, 13 (2), 47-56.

Payen D., 1983. Modélisation de l'épidémiologie de Sclerotinia sclerotiontum sur tournesol. Bull. OEPP, 13 (2), 272-281.

Perrier A., 1967. Approche thécrique de la microturbulence. La Météorologie, 4, 528-550.

Perrier A., 1976. Etude et essai de modélisation des échanges de masse et d'énergie au niveau des couverts végétaux. Thèse Doc. Etat, Univ. Paris VI, $236 \mathrm{p}$

Pinguet A., 1983. Mesure de Thumectation: transformation d'un thermohygrographe en thermohumectographe et application à une station automatique agroclimatique. Bull. OEPP, 13 (2), 43-45.

Pinter P. J., 1986. Effect of dew on canopy reflectance and temperature. Remote sensing of environment, 19, 187-205.

Rotem J., 1978. Climatic and weather influences on epidemics. In "Plant disecase" ", Vol. II. J. G. Horslall, E. B. Cowling ed., Academic Press. New York, 317-337.

Rutter A. J., Morton A. J., 1977. A predictive model of rainfall interception in forest. III. Sensitivity of the model to stand parameters and meteorological variables. J. Appl. Ecol., 14, 567-588.

Shuttleworth W. J., Wallace J. S., 1985. Evaporation from sparse crops - an energy combination theory, Quart. J. Roy. Meteorol. Soc.. $111.839-855$

Thom A. S., 1971. Momentum absorption by vegetation. Quart. J. Roy. Mercorol. Soc., 97, 414-428.

Thompson N., 1981. The duration of leaf wetness. Meteorological Magazine, I 10, 1-12

Waggoner P. E., Reifsnyder W. E., 1968. Simulation of the temperature, humidity and evaporation profiles in a leaf canopy. $J$. Appl. Meteorol. 7, 400-409.

Waggoner P. E., 1975. Micrometeorological models. In : « Vegetation and the atmosphere ", vol. 1, J. L. Monteith ed., Academic Press, New York, 205-228

Weiss A., Hagen F. A., 1983. Further experiments on the measurements of leaf wetness. Agric. Metcorol., 29, 207-212. 